\title{
Effect of Blueberry Anthocyanins Malvidin and Glycosides on the Antioxidant Properties in Endothelial Cells
}

\author{
Wuyang Huang, ${ }^{1}$ Yunming Zhu, ${ }^{1,2}$ Chunyang Li, ${ }^{1}$ Zhongquan Sui, ${ }^{3}$ and Weihong Min ${ }^{2}$ \\ ${ }^{1}$ Institute of Farm Product Processing, Jiangsu Academy of Agricultural Sciences, Nanjing 210014, China \\ ${ }^{2}$ College of Food Science and Engineering, Jilin Agricultural University, Changchun 130118, China \\ ${ }^{3}$ Department of Food Science and Engineering, School of Agriculture and Biology, Shanghai Jiao Tong University, \\ Shanghai 200240, China \\ Correspondence should be addressed to Zhongquan Sui; springsui@gmail.com and Weihong Min; minwh2000@163.com
}

Received 18 December 2015; Accepted 11 February 2016

Academic Editor: Giuseppe Cirillo

Copyright (C) 2016 Wuyang Huang et al. This is an open access article distributed under the Creative Commons Attribution License, which permits unrestricted use, distribution, and reproduction in any medium, provided the original work is properly cited.

The objective of this research was to survey the antioxidant functional role of the main anthocyanins of blueberries in endothelial cells. Changes on the reactive oxygen species (ROS), xanthine oxidase-1 (XO-1), superoxide dismutase (SOD), and heme oxygenase1 (HO-1) in cells of malvidin and the two glycosides were investigated. The results showed that these anthocyanins decreased the levels of ROS and XO-1 but increased the levels of SOD and HO-1. Glycosides improved the antioxidant capacity of malvidin to a great extent. The changes in the antioxidant properties of malvidin-3-glucoside were more pronounced than malvidin3-galactoside. Variation in levels of malvidin-3-glucoside and malvidin-3-galactoside had a significant impact on antioxidant properties to different extents. It indicates that blueberries are a good resource of anthocyanins, which can protect cells from oxidative deterioration and use blueberry as a potential functional food to prevent diseases related to oxidative stress.

\section{Introduction}

There have been intense interest and active researches in the area of dietary antioxidants to develop functional food products [1]. Rabbiteye blueberries (Vaccinium ashei) are used as endogenous antioxidant defense ingredients in the modern health-conscious food industry, due to their significant levels of anthocyanins, phenolic acids, and vitamins $[2,3]$. The health benefits of blueberries have been reported as reduction of coronary heart disease risk, visual improvement, and antimutagenic and anti-inflammatory effects [4]. The composition and molecular structure of anthocyanin determine the functional properties of blueberries [5]. Among fresh fruits and vegetables, blueberries contain greater amount of anthocyanin, especially malvidin-3-glucoside (Mv-3-glc) and malvidin-3-galactoside (Mv-3-gal) [6].

Malvidin possesses great antioxidant capacity with excellent free radical scavenging properties in vitro [7]. Malvidin exhibits antihypertensive activity by inhibiting angiotensin Iconverting enzyme and anti-inflammatory effect by blocking
NF- $\kappa$ B pathway $[8,9]$. Gopu et al. reported that malvidin interrupted quorum sensing in Klebsiella pneumoniae by docking with LasR receptor protein and reducing the violacein, EPS production, and biofilm formation [10]. In addition, malvidin plays a role in controlling both short- and longterm cellular activities. Several studies have shown that malvidin could inhibit different tumor cell lines in vitro or in vivo, including human promyelocytic/monocytic leukemia cells, gastric adenocarcinoma cells, and HT-29 colon cancer cells [11-13]. Matsunaga et al. found that malvidin could counteract oxidative stress in neuronal cells [14].

Malvidin presents in nature principally as the glycosylated form with the sugar moiety attached at position 3 on the c-ring, that is, malvidin-3-glucoside and malvidin-3galactoside. Bioavailability of anthocyanins was extensively studied due to the different mechanisms of the uptake and metabolism (deconjugation, glucuronidation, sulfation, and deglucuronidation) in various cell lines. Passamonti et al. found $\mathrm{Mv}$-3-glc possessed better bioavailability than other anthocyanidins due to the greater efficacy of binding 
to organic anion membrane carrier, bilitranslocase [15]. Rossetto et al. reported that $\mathrm{Mv}$-3-glc showed synergistic antioxidant effect with catechin on free radical-initiated peroxidation of linoleic acid in micelles [16], and Grace et al. reported its significant hypoglycemic effect in diabetic C57b1/6J mice [17]. Additionally, Mv-3-glc is a potent antiinflammatory agent in vitro and in vivo, without detectable toxicity on human peripheral blood mononuclear cells, which inhibits human macrophage-derived inflammatory mediators and decreases clinical scores in arthritic rats and inhibits ear oedema and leukocytes migration [18, 19]. Our previous study also found that $\mathrm{Mv}-3$-glc and $\mathrm{Mv}-3$ gal could inhibit TNF- $\alpha$-induced inflammatory response in endothelial cells [20]. Quintieri et al. reported that Mv-3-glc could modulate mammalian myocardial and coronary performance and protect the heart against ischemia/reperfusion injury by activating the PI3K/NO/cGMP/PKG pathway and phosphorylating AKT and eNOS [21]. Paixão et al. confirmed the capacity of $\mathrm{Mv}$-3-glc to increase NO bioavailability and to inhibit peroxynitrite-induced NF- $\kappa \mathrm{B}$ activation, supporting its benefits in cardiovascular health [22]. Anthocyanins could be as a promising tool for development of nutraceuticals to improve endothelial function. However, the antioxidant mechanisms of these anthocyanins in endothelial cells are still not clear. The objective of this study was to investigate the antioxidant functional role of the main anthocyanins in blueberries, malvidin, and its two glycosides (malvidin-3glucoside and malvidin-3-galactoside) in human umbilical vein endothelial cells.

\section{Materials and Methods}

2.1. Chemicals and Reagents. Human umbilical vein endothelial cells (HUVECs) were obtained from Zhongqiaoxinzhou Biological Technology Co., Ltd. (Shanghai, China). Dulbecco's phosphate buffer saline, malvidin, malvidin-3glucoside, malvidin-3-galactoside, and trypsin were obtained from Sigma Chemical Co., Ltd. (Nanjing, China). Fetal bovine serum and DMEM medium were obtained from Gibco/Invitrogen (Shanghai, China). Streptomycin and penicillin were obtained from Life Technologies (Shanghai, China). ROS Assay Kit was obtained from Biyotime Institute of Biotechnology (Shanghai, China). XO-1, SOD, and HO1 ELISA Kit were obtained from Boster Biotechnology Inc. (Wuhan, China). All chemicals and reagents are of analytical grade.

2.2. Antibodies. Rabbit monoclonal primary antibody against $\mathrm{XDH}$, mouse polyclonal primary antibodies to the $\beta$-actin, and goat anti-rabbit/mouse IgG-HRP conjugated secondary antibody were obtained from Boster Biotechnology Inc. (Wuhan, China). Primary antibodies against XDH were used at 1:400 dilutions, and primary antibodies against $\beta$-actin were used at 1:1000 dilutions. Secondary antibodies were used at $1: 2000$ dilutions.

2.3. Endothelial Cell Culture and Treatment. HUVECs could be characterized as a model system for studying oxidative stress in the vasculature. The cells were cultured according to a laboratory protocol [20]. After quiescing in a reduced serum medium for $4 \mathrm{~h}$, the cells were treated with 1,5 , and $10 \mu \mathrm{mol} / \mathrm{L}$ of $\mathrm{Mv}, \mathrm{Mv}$-glc, and Mv-gal, or mixture of Mv-glc and Mv-gal for $24 \mathrm{~h}$, respectively. DMSO was instead in the control.

2.4. Reactive Oxygen Species (ROS) Assay. Dichloro-dihydrofluorescein diacetate (DCFH-DA) assay is a quantitative method for oxidative stress assessment in cells. In this study, DCFH-DA detection kit was used to assess the ROS level in endothelial cells. Briefly, the cells were seeded in 6-well plates, treated with different samples to incubate for $24 \mathrm{~h}$. After washing cells with PBS, $10 \mu \mathrm{mol} / \mathrm{L}$ DCFH-DA was added to each well and reacted for $20 \mathrm{~min}$ at $37^{\circ} \mathrm{C}$, and then the cells were washed thoroughly with PBS. A group of cells was visualized under an IX53 Inverted Fluorescent Microscope (Olympus, Tokyo, Japan) at $530 \mathrm{~nm}$ emission and $485 \mathrm{~nm}$ excitation filters immediately. All images presented are in $\times 200$ magnification. Another one was collected in $1 \mathrm{~mL}$ PBS after dissociated, and fluorescence was recorded by LB 941 TriStar Microplate Reader (Berthold Technologies, Bad Wildbad, Germany). The total fluorescence intensity of cells in each well was noted, and ROS generation was measured as fold increase over the untreated control.

2.5. ELISA Analysis and Western Blotting. The levels of XO$1, \mathrm{SOD}$, and $\mathrm{HO}-1$ in the supernatants were detected using ELISA kits according to the kit protocol booklet instructions. The absorbance was measured at $450 \mathrm{~nm}$ on a StatFax-2100 Microplate Reader (Awareness Technology Inc., Plam, FL, USA) via Hyper Terminal Applet ELISA software.

$\mathrm{XO}-1$ protein expression was also analyzed by western blotting performed on the HUVEC lysates. $\beta$-actin was used as a loading control. All data were expressed as fold increase over the untreated control.

2.6. Statistical Analysis. Data were expressed as mean value \pm standard deviation (SD) of triplicate determinations. Figures were obtained using GraphPad Prism Version 5 (GraphPad Software, Inc., CA, USA). One-way ANOVA was used for the determination of statistical significance using SPSS 19.0 Software. The differences were considered statistically significant with a $P$ value of 0.05 .

\section{Results}

3.1. Effects of Malvidin and Its Glycosides on Reactive Oxygen Species in Cells. Addition of all of the tested malvidin, malvidin-3-glucoside, malvidin-3-galactoside, and the mixture of the two glycosides decreased ROS values in endothelial cells (Figure 1). Malvidin treated at 1,5 , and $10 \mu \mathrm{mol} / \mathrm{L}$ concentration inhibited $4 \%, 6 \%$, and $11 \%$ ROS, respectively. When Mv-3-glc was present, the values for ROS production decreased $21 \%, 35 \%$, and $28 \%$ at the concentration of 1 , 5 , and $10 \mu \mathrm{mol} / \mathrm{L}$, respectively, whereas when $\mathrm{Mv}$-3-gal was present, the values decreased $12 \%, 8 \%$, and $9 \%$, respectively. The mixture of Mv-3-glc and Mv-3-gal significantly decreased ROS level in cells. Both Mv-3-glc and the mixture of 


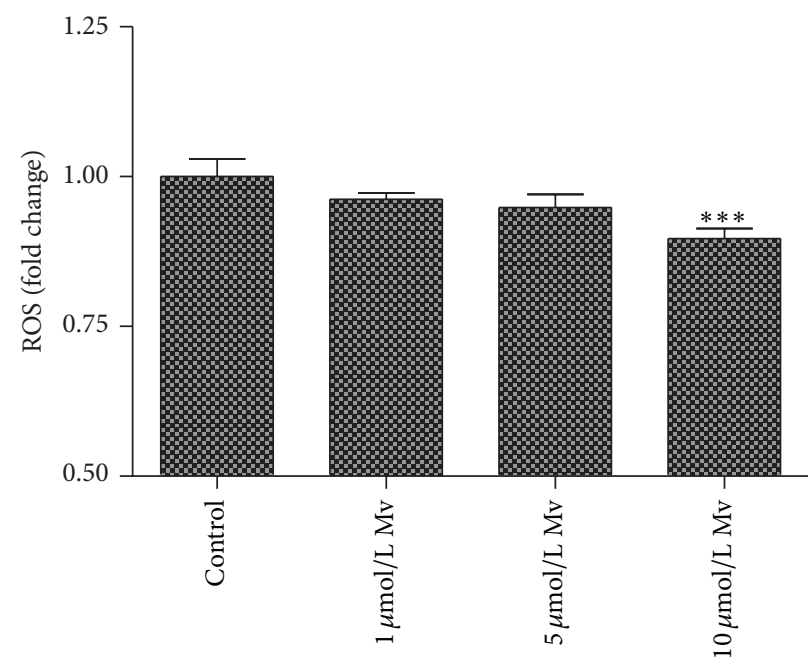

(a)

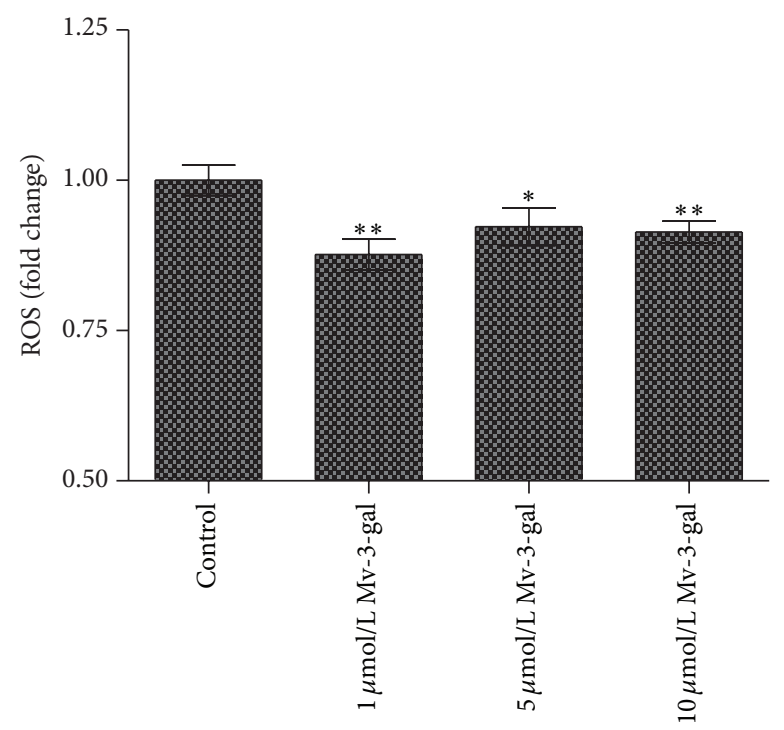

(c)

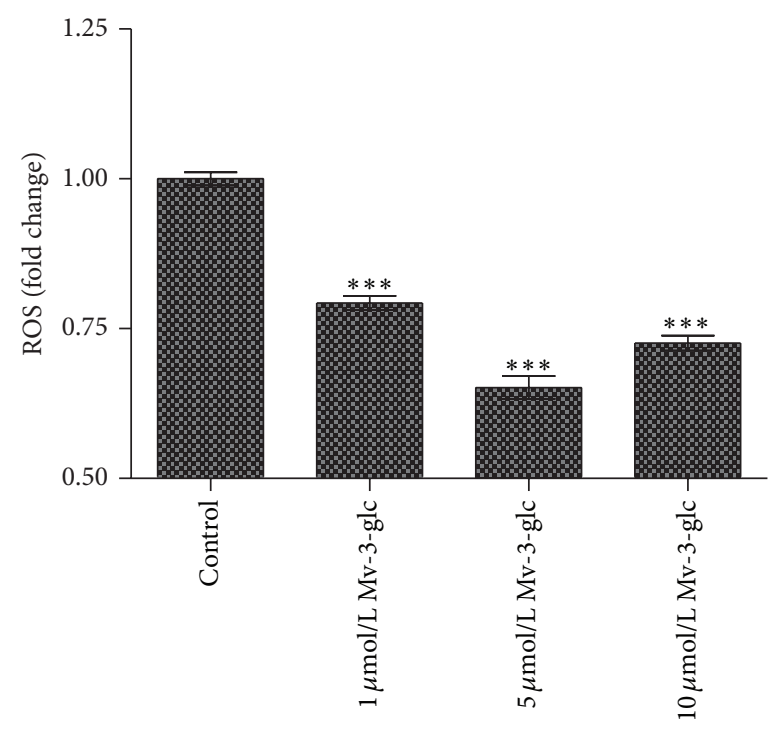

(b)

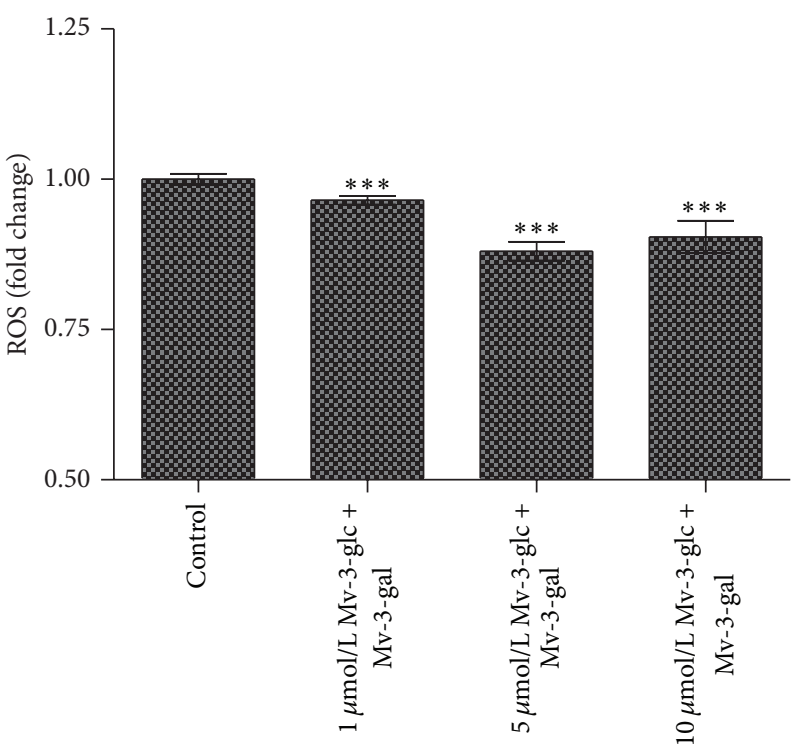

(d)

FiguRE 1: Effects of different concentrations Mv (a), Mv-3-glc (b), Mv-3-gal (c), and Mv-3-glc + Mv-3-gal (d) on the level of ROS in HUVECs. $*, * *$, and $* * *$ indicate $P<0.05, P<0.01$, and $P<0.001$, respectively, compared to the control.

the two glycosides produced the greatest inhibition rate at the concentration of $5 \mu \mathrm{mol} / \mathrm{L}$, whereas $\mathrm{Mv}$-3-gal produced the largest inhibition rate at the concentration of $1 \mu \mathrm{mol} / \mathrm{L}$. The extent of the decrease in ROS of Mv-3-glc was more pronounced than that of Mv-3-gal. However, there seemed to be an antagonism effect between Mv-3-glc and Mv-3-gal, since the mixture possessed lower ROS scavenging activity than Mv-3-glc. The fluorescence intensity levels of ROS showed similar effects (Figure 2).

\subsection{Effects of Malvidin and Its Glycosides on XO-1 Production} in Supernatant and Cells. Addition of all the tested Mv, Mv-3glc, Mv-3-gal, and the mixture decreased XO-1 production in supernatant (Figure 3). When the values of $\mathrm{XO}-1$ production in supernatant treated with 1,5 , and $10 \mu \mathrm{mol} / \mathrm{L}$ malvidin were compared to those of the control, they were found to be $0.68,0.76$, and 0.92 times, respectively. Interestingly, only $\mathrm{Mv}$ treated at lower concentration $(1 \mu \mathrm{mol} / \mathrm{L})$ had significant inhibitory effect on XO-1 protein. The cells treated with 1 , 5, and $10 \mu \mathrm{mol} / \mathrm{L} \mathrm{Mv-3-glc,} \mathrm{Mv-3-gal,} \mathrm{and} \mathrm{the} \mathrm{mixture} \mathrm{all}$ significantly inhibited XO-1 level at the rate of $15 \%, 19 \%$, $51 \%$, and $13 \%, 23 \%, 31 \%$, and $19 \%, 29 \%, 5 \%$, respectively. The two malvidin glycosides exhibited greater inhibitory effect than malvidin, whereas Mv-3-glc showed stronger effect than Mv-3-gal in most cases. Mv-3-glc at the concentration of $10 \mu \mathrm{mol} / \mathrm{L}$ reached the greatest inhibition rate. Consistent with ROS results, Mv-3-glc had antagonistic effect with Mv3 -gal at high concentration $(10 \mu \mathrm{mol} / \mathrm{L})$. 

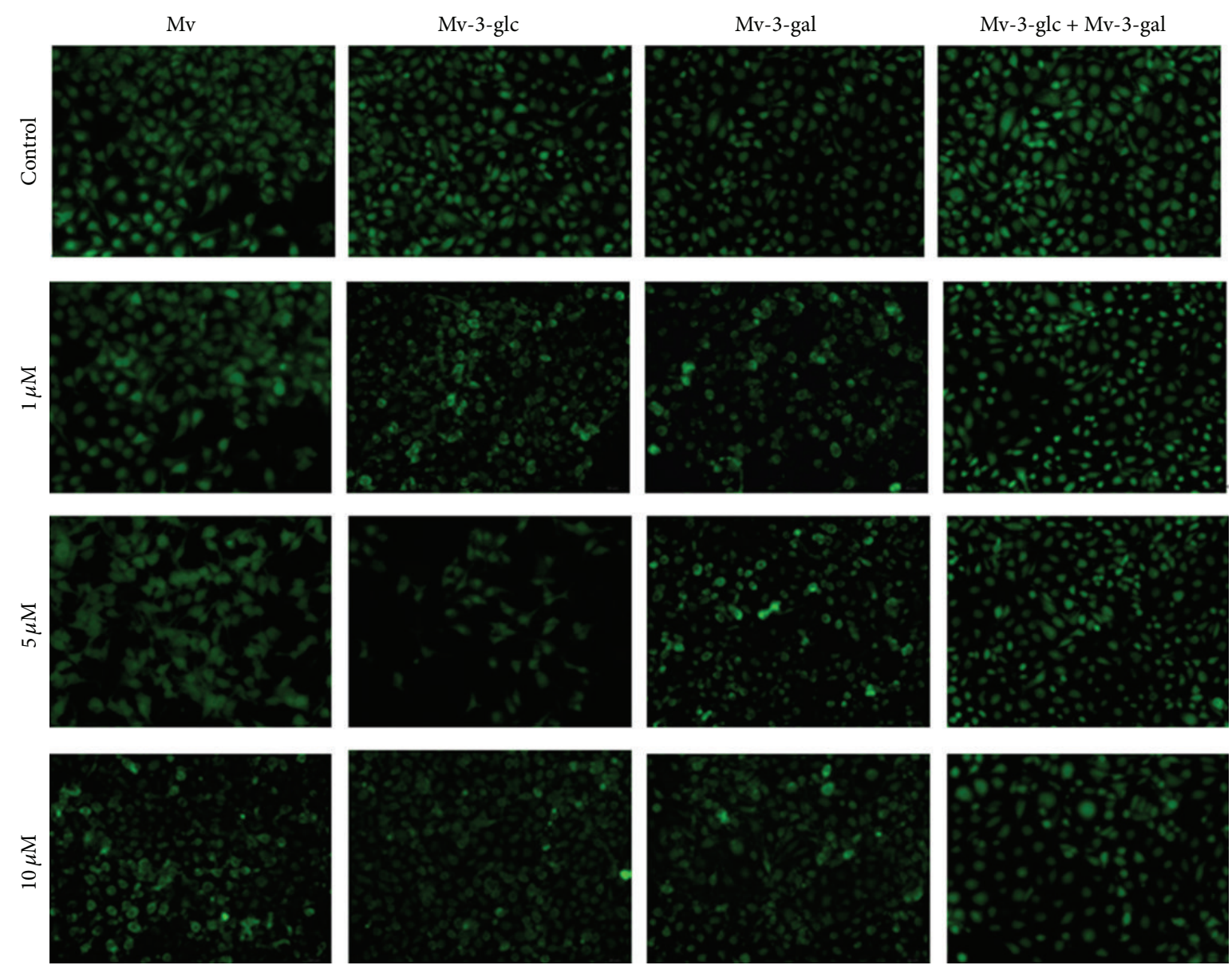

FIgURE 2: The fluorescence intensity of ROS in HUVECs treated with different concentrations Mv, Mv-3-glc, Mv-3-gal, and Mv-3-glc + Mv-3-gal. A representative set of images from three independent experiments is shown. All images presented are in $\times 200$ magnification.

Addition of Mv, Mv-3-glc, Mv-3-gal, and the mixture also decreased the values of $\mathrm{XO}-1$ production in cells to different extents (Figure 4$)$. Low concentration of $\mathrm{Mv}(1$ and $5 \mu \mathrm{mol} / \mathrm{L})$ had significant inhibitory effects on endothelial XO-1 protein. The relative contents (XO- $1 / \beta$-actin) were 0.88 and 0.86 times compared to those of the control, respectively. The XO-1 relative contents in the cells treated with 1,5 , and $10 \mu \mathrm{mol} / \mathrm{L} \mathrm{Mv-}$ 3-glc, Mv-3-gal, and the mixture were 0.91, 0.74, 0.87 and 0.95, $0.79,0.81$, and $0.83,0.76,0.93$ times than those of the control, respectively. High concentration of the mixture $(10 \mu \mathrm{mol} / \mathrm{L})$ had no effect on the XO-1 protein expression in cells, whereas $\mathrm{Mv}-3$-glc tended to produce greater inhibition rate.

3.3. Effects of Malvidin and Its Glycosides on SOD Production in Supernatant. Malvidin increased the level of SOD production as a function of concentration. When the values of SOD production of the cells treated with 1,5 , and $10 \mu \mathrm{mol} / \mathrm{L}$ malvidin were compared to those of the control, they were found to be 1.64, 2.07, and 2.27 times, respectively. Addition of $\mathrm{Mv}$-3-glc and $\mathrm{Mv}$-3-gal greatly increased SOD production in supernatant. When the SOD production for Mv-3-glc and
Mv-3-gal treated at the concentration of 1,5 , and $10 \mu \mathrm{mol} / \mathrm{L}$ was compared to those of the control, they were found to be 3.26, 4.79, 2.59 and 3.06, 2.01, 1.90 times, respectively. However, all products of the mixture of $\mathrm{Mv}$-3-glc and Mv-3-gal at different concentration showed less effect, with changes over the control at 1.55, 1.81, and 1.37 times, respectively (Figure 5).

3.4. Effects of Malvidin and Its Glycosides on HO-1 Production in Supernatant. The control contained $2.09 \mu \mathrm{g} / \mathrm{L}$ HO-1, while the cells treated with 1,5 , and $10 \mu \mathrm{mol} / \mathrm{L}$ malvidin contained $2.59 \mu \mathrm{g} / \mathrm{L}, 2.84 \mu \mathrm{g} / \mathrm{L}$, and $2.24 \mu \mathrm{g} / \mathrm{L} \mathrm{HO}-1$, which were 1.24 , 1.36 , and 1.08 times than those of the control. The supernatant HO-1 levels of the cells treated with 1,5 , and $10 \mu \mathrm{mol} / \mathrm{L} \mathrm{Mv-}$ 3-glc, Mv-3-gal, and the mixture were 1.25, 1.38, 1.18, and $1.05,1.38,1.23$, and $1.67,2.03,1.24$ times than those of the control, respectively (Figure 6). Addition of malvidin and its glycosides produced a variety of effects on $\mathrm{HO}-1$ production, but they all reached the greatest values at the concentration of $5 \mu \mathrm{mol} / \mathrm{L}$. There were synergistic effects between $\mathrm{Mv}-3$ glc and Mv-3-gal, since the mixtures at all the concentration showed greater increase rates than the others. 


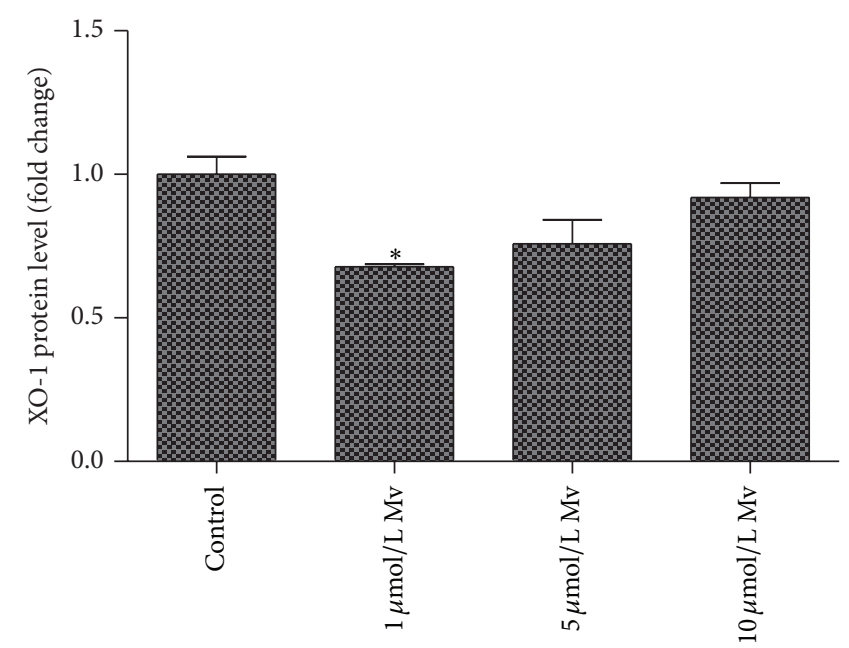

(a)

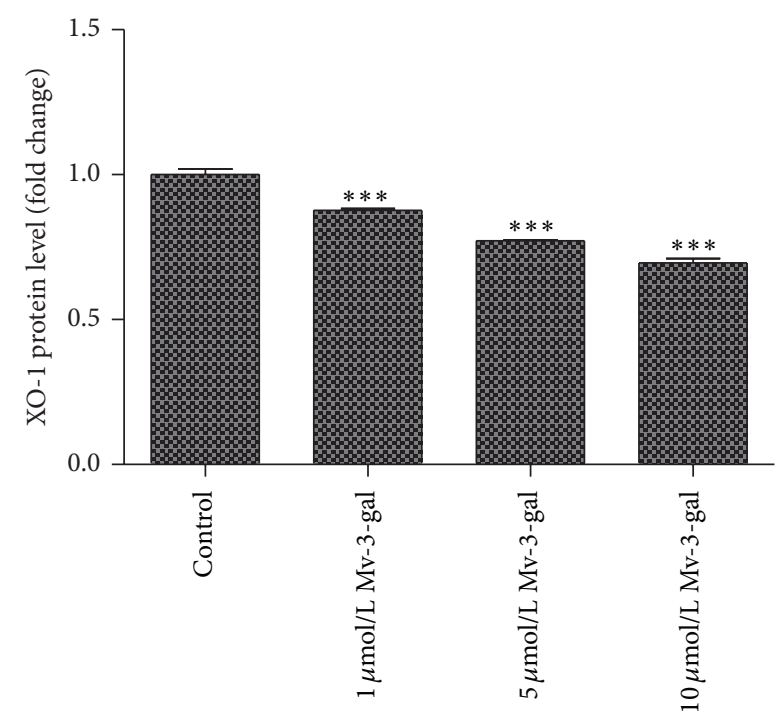

(c)

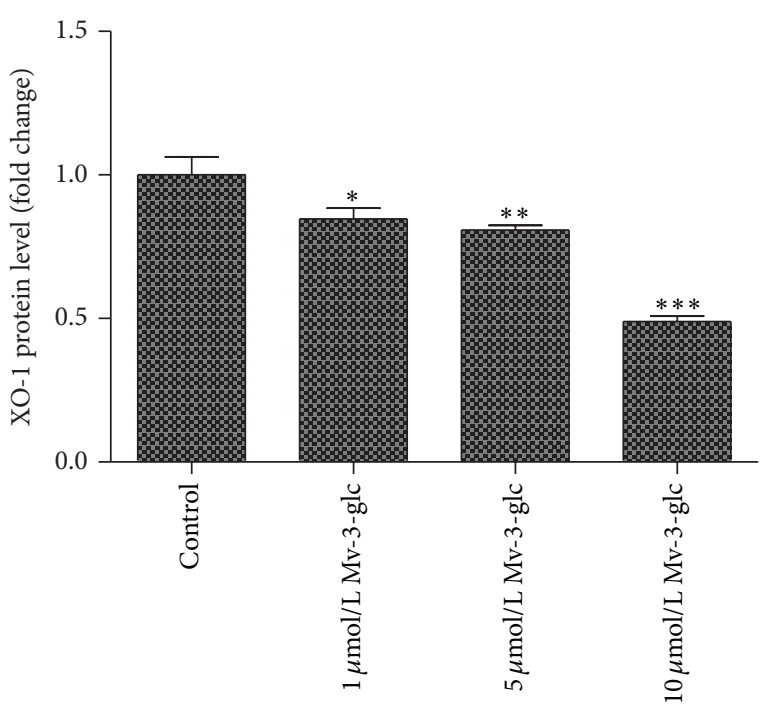

(b)

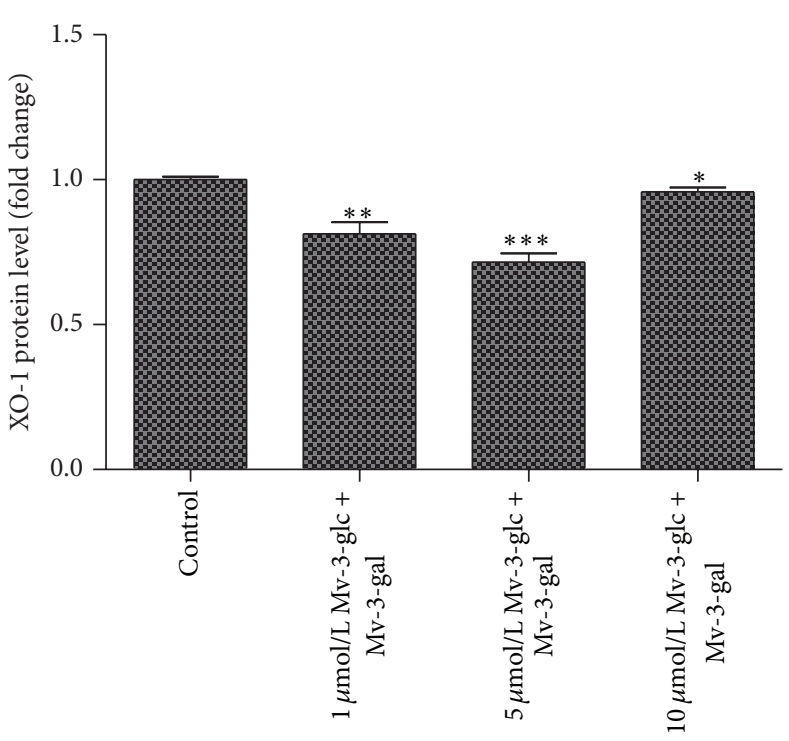

(d)

Figure 3: Effects of different concentrations Mv (a), Mv-3-glc (b), Mv-3-gal (c), and Mv-3-glc + Mv-3-gal (d) on XO-1 production released into the supernatant. $*, * *$, and $* * *$ indicate $P<0.05, P<0.01$, and $P<0.001$, respectively, compared to the control.

\section{Discussion}

The molecular structure and concentration of anthocyanins in berries determine their bioactivities. Anthocyanins have peculiar chemical structures deficient in electron [23]. The efficacy to scavenge diverse ROS differs from one to another. Generally the antioxidant capacity of anthocyanins is associated with the number of free hydroxyls around the pyrone ring, but this is not always true [24]. Different parameters, including bond dissociation enthalpy, electron transfer enthalpy, electrophilicity, frontier charge density, hardness, ionization potential, and proton affinity, should be calculated to evaluate anthocyanin antioxidant characteristics [7]. A density functional theory study found that antioxidant capacity of different anthocyanin structures was in the following order: cyanidin $>$ malvidin $>$ aurantinidin $>$ delphinidin $>$ peonidin $>$ pelargonidin [25]. Blueberries are known to contain a significant level of anthocyanins, in which malvidin3-galactoside and malvidin-3-glucoside are the most dominant [6]. Malvidin has four hydroxyls, leading to a good antioxidant capacity. Therefore, blueberries exhibited obvious superiority in the prevention of chronic diseases caused by oxidative damage.

Antioxidants have been shown to delay, inhibit, and prevent the oxidation by interacting with biological systems through many potential mechanisms, such as absorbing oxygen radicals, chelating of the metal ions, scavenging free radicals, regulating enzyme activity and protein levels, and blocking signaling pathways [26]. In the present study, blueberry anthocyanins, malvidin, and its glycosides could 


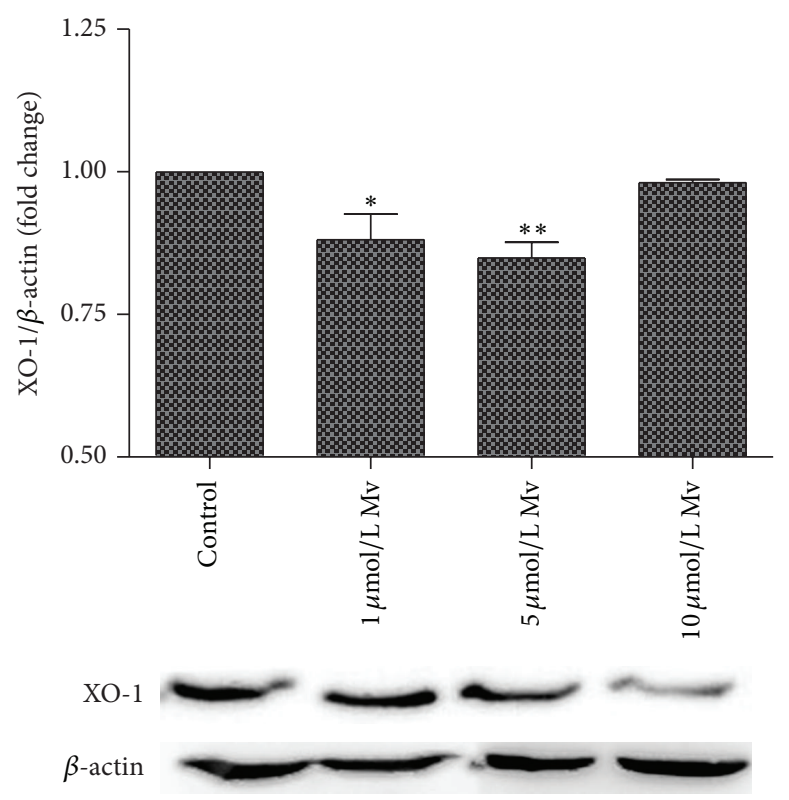

(a)

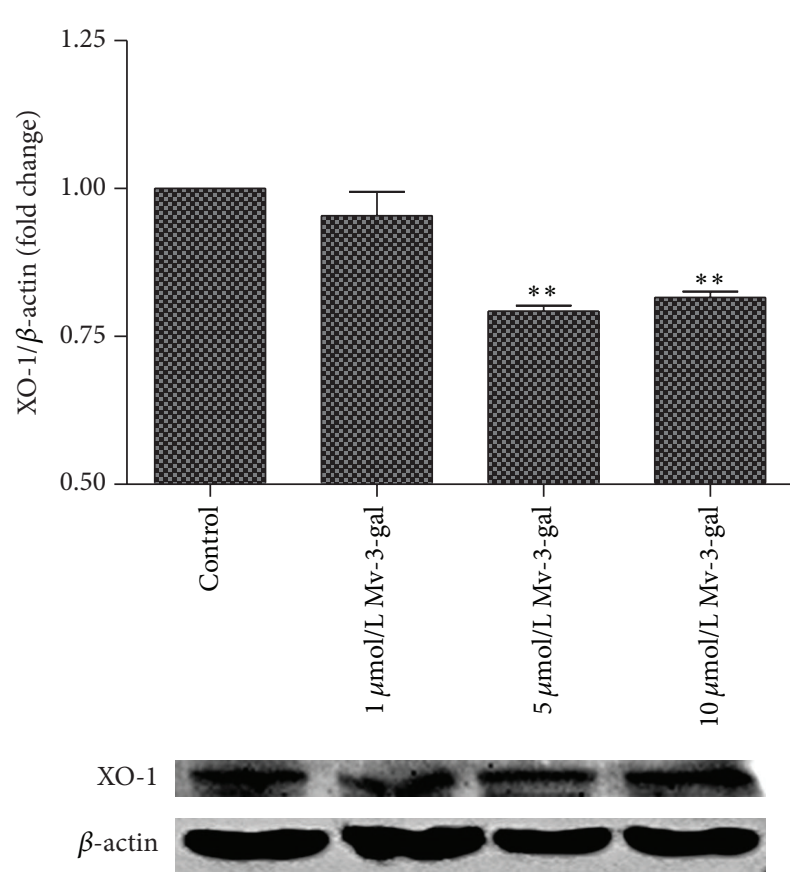

(c)

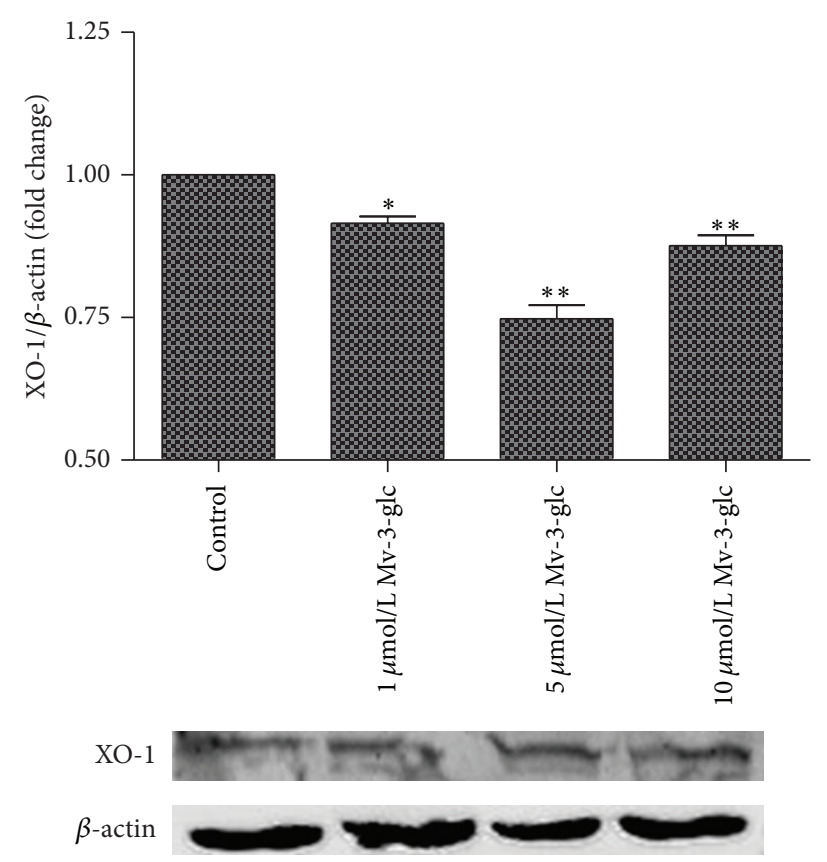

(b)

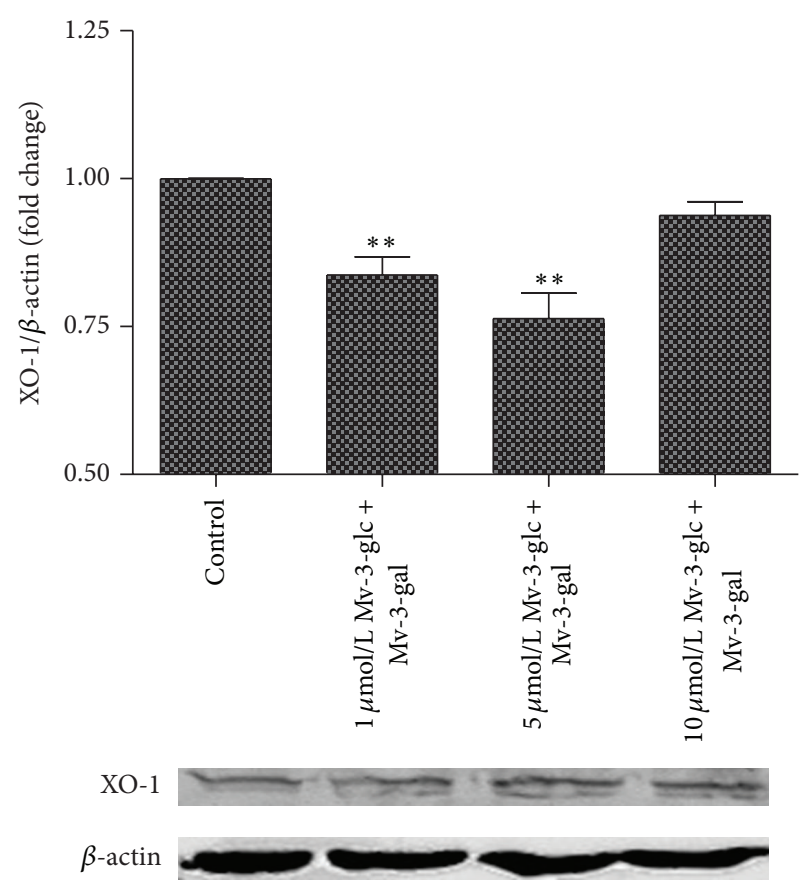

(d)

Figure 4: Effects of different concentrations Mv (a), Mv-3-glc (b), Mv-3-gal (c), and Mv-3-glc + Mv-3-gal (d) on XO-1 production in the cells. $*$ and $* *$ indicate $P<0.05$ and $P<0.01$, respectively, compared to the control.

reduce oxidative stress and alleviate harmful effects by greatly decreasing the level of ROS in endothelial cells, as well as changing several key proteins' levels. Xanthine oxidase-1 (XO-1), a major source of superoxide, has been implicated in endothelial dysfunction partly due to the rapid inactivation of nitric oxide [27]. Superoxide dismutase (SOD) and heme oxygenase-1 (HO-1) are both the important antioxidant defense against endothelial oxidative damage [28, 29]. The capacity of Mv, Mv-3-glc, Mv-3-gal, and the glycoside mixture on decreasing XO protein level and increasing SOD and HO-1 protein levels further confirmed that blueberry anthocyanins are a potential source of antioxidants. 


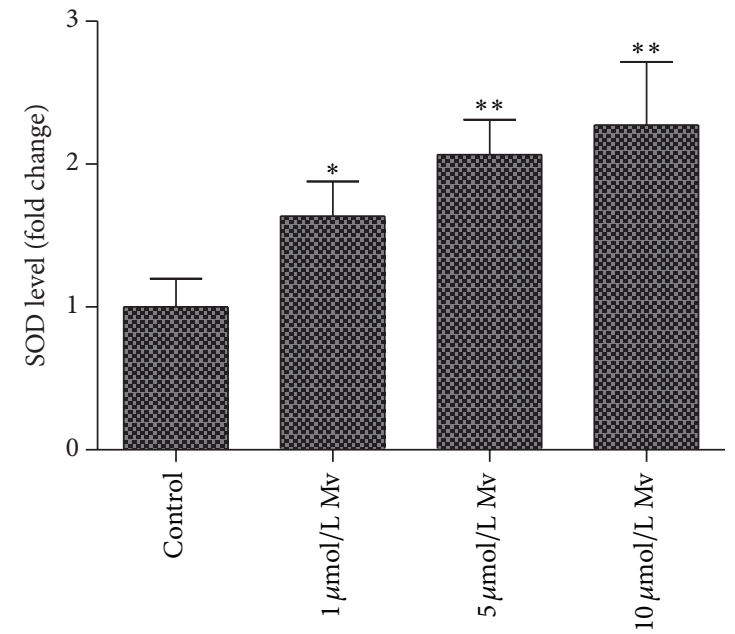

(a)

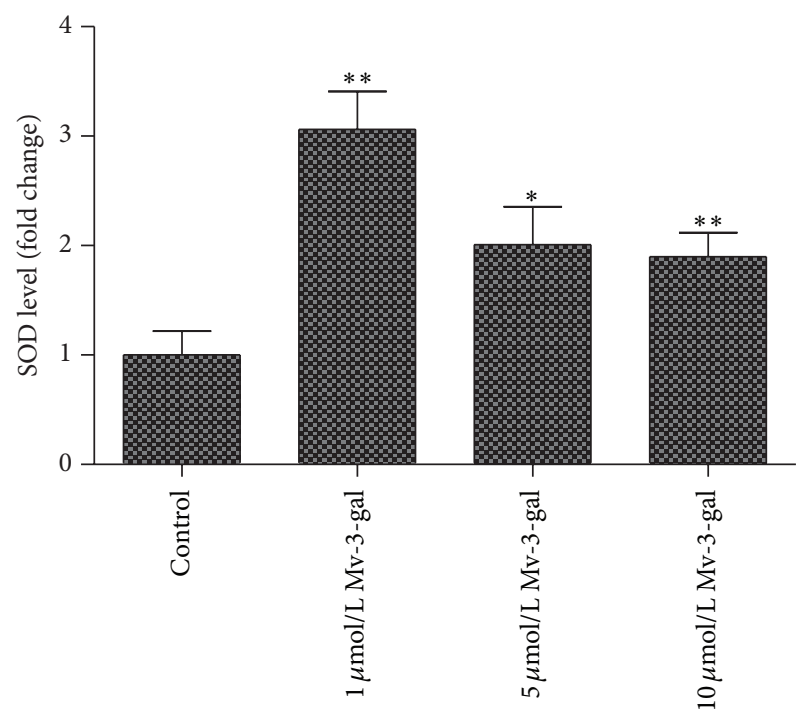

(c)

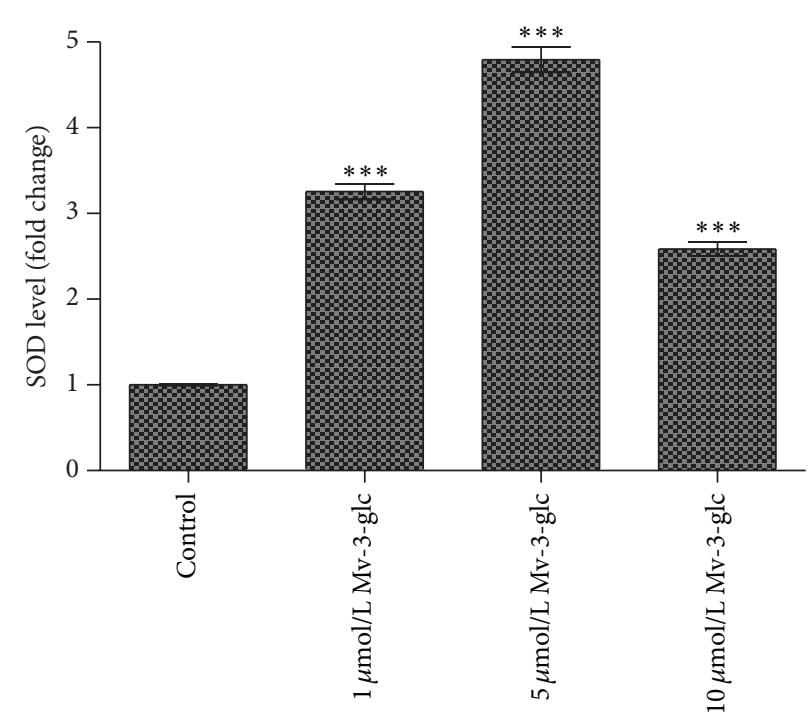

(b)

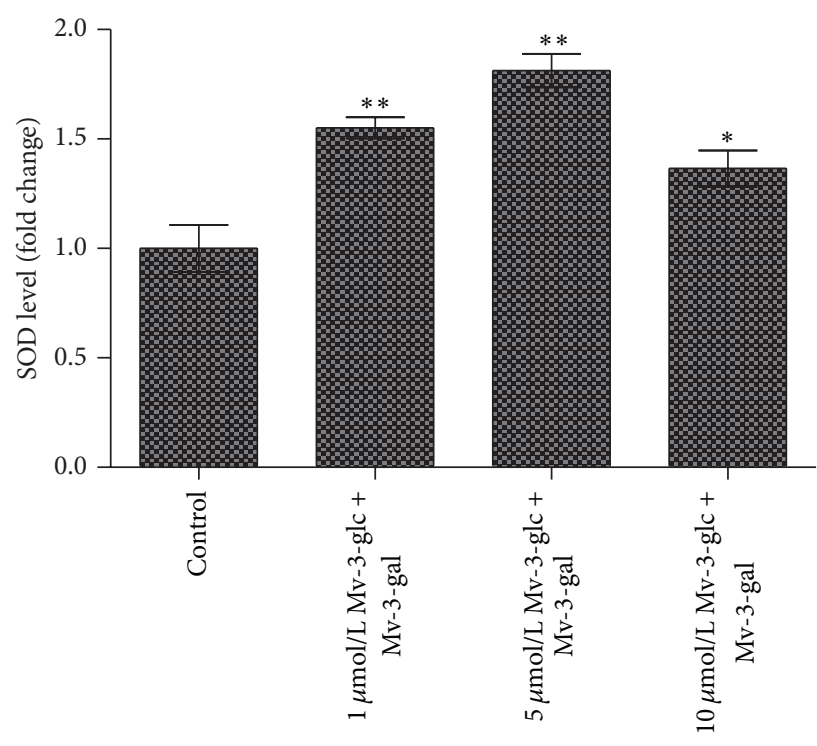

(d)

FIGURE 5: Effects of different concentrations Mv (a), Mv-3-glc (b), Mv-3-gal (c), and Mv-3-glc + Mv-3-gal (d) on SOD level in the supernatant. $*, * *$, and $* * *$ indicate $P<0.05, P<0.01$, and $P<0.001$, respectively, compared to the control.

Generally glycosylation of an anthocyanin seems to decrease the antioxidant capacity compared with the aglycone because it reduces free hydroxyls and metal chelation sites [30]. However, Kähkönen and Heinonen found that different glycosylation patterns either enhanced or diminished the antioxidant power depending on the anthocyanidin and models used for antioxidant analysis because the in vitro effect of glycosylation on antioxidant activity depended on the environment when oxidations occurred [31]. Fukumoto and Mazza reported that antioxidant activity increased with hydroxyl groups but decreased with glycosylation of anthocyanidins. Interestingly, this study showed that malvidin-3-glucoside and malvidin-3-galactoside had better antioxidant capacity than malvidin in endothelial cells [32]. In addition, glucoside seemed to be more effective than galactoside on antioxidant improvement. It further confirmed that the molecular structure of glucosylation was the most important factor in determining antioxidant properties of anthocyanins [24]. The results showed that the typical major dietary anthocyanins in blueberries, Mv-3-glc and Mv-3gal, had great antioxidant properties in endothelial cells. It indicated that they could protect cells from oxidative deterioration and used as a potential functional food ingredients to prevent diseases related to oxidative stress. 


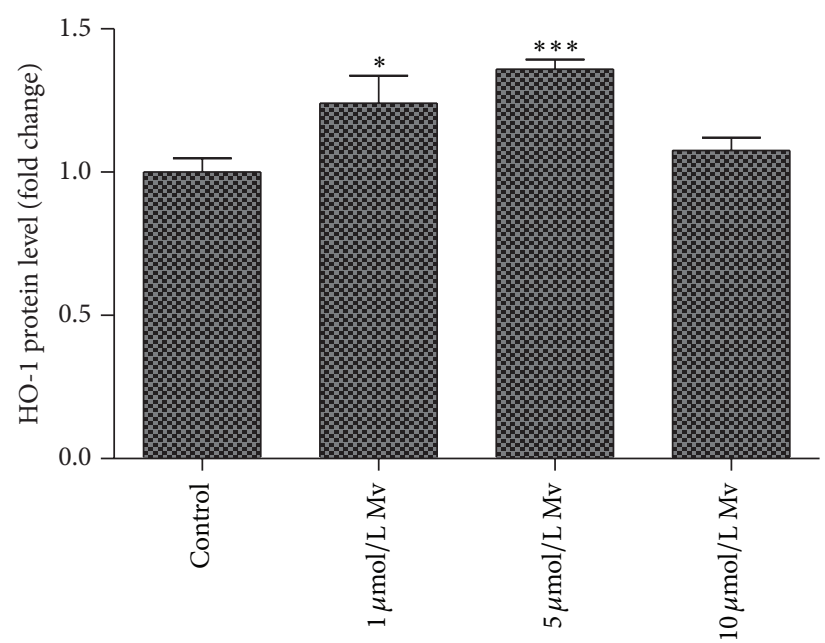

(a)

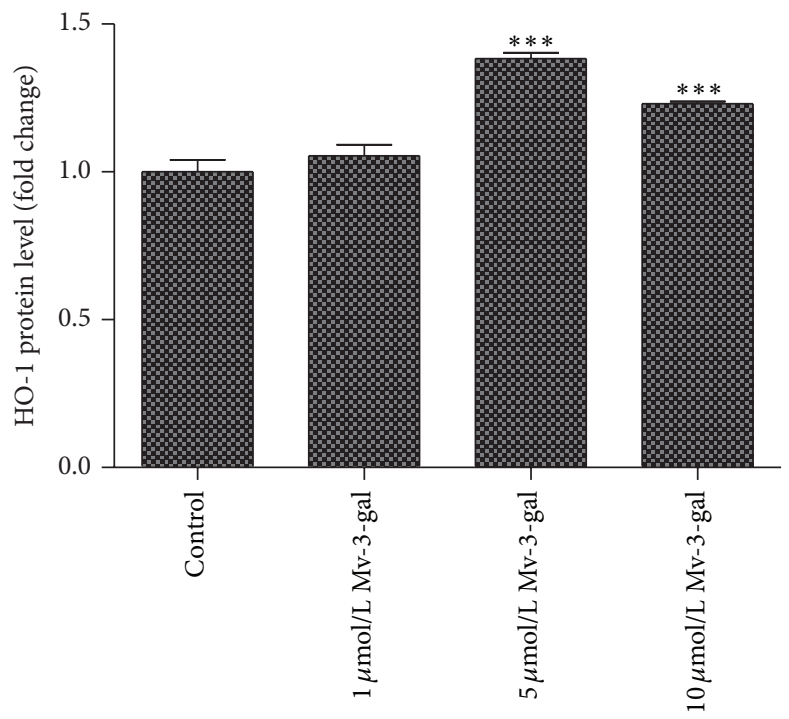

(c)

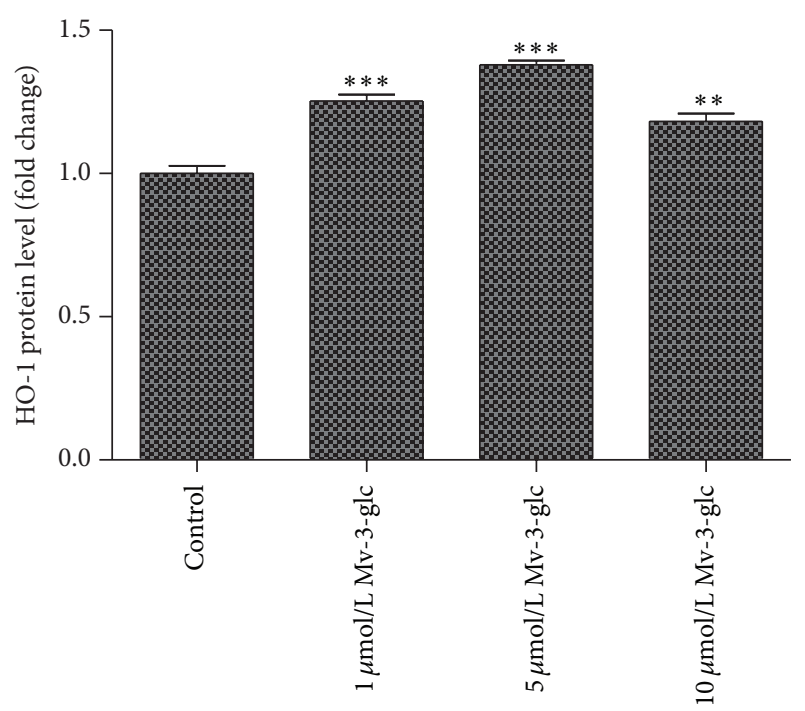

(b)

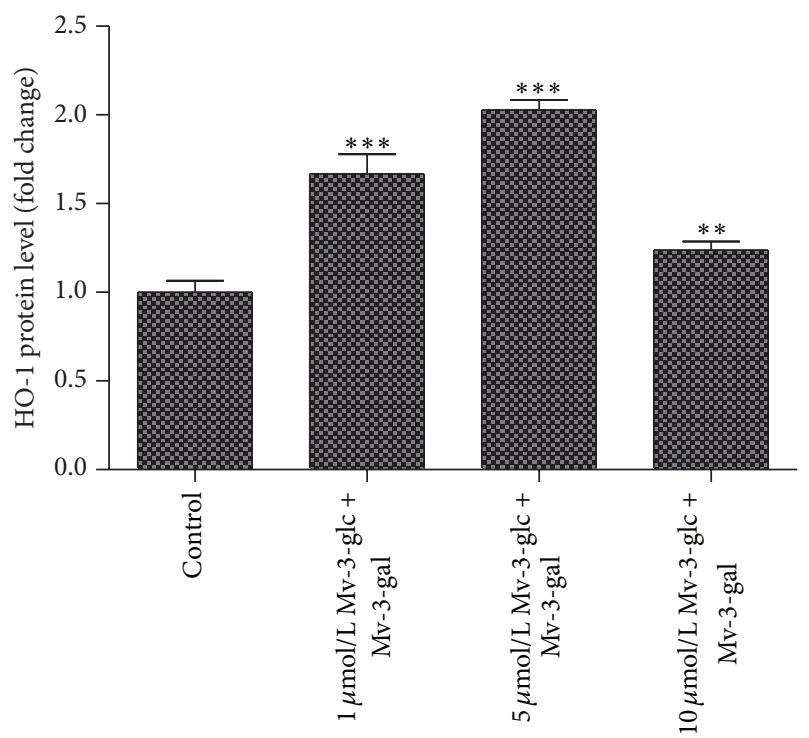

(d)

Figure 6: Effects of different concentrations Mv (a), Mv-3-glc (b), Mv-3-gal (c), and Mv-3-glc + Mv-3-gal (d) on HO-1 production released into the supernatant. $*, * *$, and $* * *$ indicate $P<0.05, P<0.01$, and $P<0.001$, respectively, compared to the control.

\section{Conclusions}

In the present study, treatment with malvidin, malvidin-3glucoside, malvidin-3-galactoside, and the mixture of the two glycosides significantly attenuated oxidative stress in human umbilical vein endothelial cells. Mv, Mv-3-glc, Mv-3-gal, and the mixture all showed good antioxidant capacity in cells by the mechanism of inhibiting ROS and $\mathrm{XO}-1$ levels and increasing the SOD and HO-1 levels. In most cases, Mv-3glc had better potential antioxidant effect than Mv-3-gal. This indicated that bioactive anthocyanins in blueberries, such as $\mathrm{Mv}$-3-glc and Mv-3-gal, could be applied in the production of smart and innovative pharmaceutical or functional food ingredients to improve endothelial function and prevent the progression of diseases caused by oxidative stress.

\section{Conflict of Interests}

There is no conflict of interests regarding the publication of this paper.

\section{Acknowledgments}

This research was supported by grants from National Natural Science Foundation of China (NSFC31101264; 31301419) and 
Natural Science Foundation Program of Jiangsu Province (BK20141386).

\section{References}

[1] I. R. Astadi, M. Astuti, U. Santoso, and P. S. Nugraheni, "In vitro antioxidant activity of anthocyanins of black soybean seed coat in human low density lipoprotein (LDL)," Food Chemistry, vol. 112, no. 3, pp. 659-663, 2009.

[2] V. Dragović-Uzelac, Z. Savić, A. Brala, B. Levaj, D. B. Kovaćević, and A. Biško, "Evaluation of phenolic content and antioxidant capacity of blueberry cultivars (Vaccinium corymbosum L.) grown in the Northwest Croatia," Food Technology and Biotechnology, vol. 48, no. 2, pp. 214-221, 2010.

[3] E. Pojer, F. Mattivi, D. Johnson, and C. S. Stockley, "The case for anthocyanin consumption to promote human health: a review," Comprehensive Reviews in Food Science and Food Safety, vol. 12, no. 5, pp. 483-508, 2013.

[4] J. Chen, Y. Zhao, X.-Y. Tao, M. Zhang, and A.-D. Sun, "Protective effect of blueberry anthocyanins in a CCL4-induced liver cell model," LWT_Food Science and Technology, vol. 60, no. 2, pp. 1105-1112, 2015.

[5] S. Y. Wang, H. Chen, M. J. Camp, and M. K. Ehlenfeldt, "Genotype and growing season influence blueberry antioxidant capacity and other quality attributes," International Journal of Food Science and Technology, vol. 47, no. 7, pp. 1540-1549, 2012.

[6] C. Li, J. Feng, W.-Y. Huang, and X.-T. An, "Composition of polyphenols and antioxidant activity of rabbiteye blueberry (Vaccinium ashei) in Nanjing," Journal of Agricultural and Food Chemistry, vol. 61, no. 3, pp. 523-531, 2013.

[7] R. Pop, M. N. Ştefănut, A. Căta, C. Tănasie, and M. Medeleanu, "Ab initio study regarding the evaluation of the antioxidant character of cyanidin, delphinidin and malvidin," Central European Journal of Chemistry, vol. 10, no. 1, pp. 180-186, 2012.

[8] C. Lee, D. Han, B. Kim, N. Baek, and B.-K. Baik, "Antioxidant and anti-hypertensive activity of anthocyanin-rich extracts from hulless pigmented barley cultivars," International Journal of Food Science and Technology, vol. 48, no. 5, pp. 984-991, 2013.

[9] W.-Y. Huang, J. Wang, Y.-M. Liu, Q.-S. Zheng, and C.-Y. Li, "Inhibitory effect of Malvidin on TNF- $\alpha$-induced inflammatory response in endothelial cells," European Journal of Pharmacology, vol. 723, no. 1, pp. 67-72, 2014.

[10] V. Gopu, S. Kothandapani, and P. H. Shetty, "Quorum quenching activity of Syzygium cumini (L.) Skeels and its anthocyanin malvidin against Klebsiella pneumoniae," Microbial Pathogenesis, vol. 79, no. 1, pp. 61-69, 2015.

[11] J. W. Hyun and H. S. Chung, "Cyanidin and malvidin from Oryza sativa cv. Heugjinjubyeo mediate cytotoxicity against human monocytic leukemia cells by arrest of $\mathrm{G}_{2} / \mathrm{M}$ phase and induction of apoptosis," Journal of Agricultural and Food Chemistry, vol. 52, no. 8, pp. 2213-2217, 2004.

[12] P.-H. Shih, C.-T. Yeh, and G.-C. Yen, "Effects of anthocyanidin on the inhibition of proliferation and induction of apoptosis in human gastric adenocarcinoma cells," Food and Chemical Toxicology, vol. 43, no. 10, pp. 1557-1566, 2005.

[13] S. J. Patterson, J. G. Fischer, and R. V. Dulebohn, "DNA damage in HT-29 colon cancer cells is enhanced by high concentrations of the anthocyanin malvidin," The FASEB Journal, vol. 22, pp. 890-900, 2008.

[14] N. Matsunaga, S. Imai, Y. Inokuchi et al., "Bilberry and its main constituents have neuroprotective effects against retinal neuronal damage in vitro and in vivo," Molecular Nutrition and Food Research, vol. 53, no. 7, pp. 869-877, 2009.

[15] S. Passamonti, U. Vrhovsek, and F. Mattivi, "The interaction of anthocyanins with bilitranslocase," Biochemical and Biophysical Research Communications, vol. 296, no. 3, pp. 631-636, 2002.

[16] M. Rossetto, P. Vanzani, F. Mattivi, M. Lunelli, M. Scarpa, and A. Rigo, "Synergistic antioxidant effect of catechin and malvidin-3glucoside on free radical-initiated peroxidation of linoleic acid in micelles," Archives of Biochemistry and Biophysics, vol. 408, no. 2, pp. 239-245, 2002.

[17] M. H. Grace, D. M. Ribnicky, P. Kuhn et al., "Hypoglycemic activity of a novel anthocyanin-rich formulation from lowbush blueberry, Vaccinium angustifolium Aiton," Phytomedicine, vol. 16, no. 5, pp. 406-415, 2009.

[18] A. S. Decendit, M. Mamani-Matsuda, V. Aumont et al., "Malvidin-3-O- $\beta$-glucoside, major grape anthocyanin, inhibits human macrophage-derived inflammatory mediators and decreases clinical scores in arthritic rats," Biochemical Pharmacology, vol. 86, no. 10, pp. 1461-1467, 2013.

[19] A. S. Prudente, A. M. V. Loddi, M. R. Duarte et al., "Pre-clinical anti-inflammatory aspects of a cuisine and medicinal millennial herb: Malva sylvestris L.", Food and Chemical Toxicology, vol. 58, pp. 324-331, 2013.

[20] W.-Y. Huang, Y.-M. Liu, J. Wang, X.-N. Wang, and C.-Y. $\mathrm{Li}$, "Anti-inflammatory effect of the blueberry anthocyanins malvidin-3-glucoside and malvidin-3-galactoside in endothelial cells," Molecules, vol. 19, no. 8, pp. 12827-12841, 2014.

[21] A. M. Quintieri, N. Baldino, E. Filice et al., "Malvidin, a red wine polyphenol, modulates mammalian myocardial and coronary performance and protects the heart against ischemia/reperfusion injury," Journal of Nutritional Biochemistry, vol. 24, no. 7, pp. 1221-1231, 2013.

[22] J. Paixão, T. C. P. Dinis, and L. M. Almeida, "Malvidin-3glucoside protects endothelial cells up-regulating endothelial NO synthase and inhibiting peroxynitrite-induced NF-kB activation," Chemico-Biological Interactions, vol. 199, no. 3, pp. 192200, 2012.

[23] J. B. Harborne and C. A. Williams, "Advances in flavonoid research since 1992," Phytochemistry, vol. 55, no. 6, pp. 481-504, 2000 .

[24] S. Lucioli, "Anthocyanins: mechanism of action and therapeutic efficacy," in Medicinal Plants as Antioxidant Agents: Understanding Their Mechanism of Action and Therapeutic Efficacy, A. Capasso, Ed., pp. 27-57, Research Signpost, Kerala, India, 2012.

[25] R. Guzmán, C. Santiago, and M. Sánchez, "A density functional study of antioxidant properties on anthocyanidins," Journal of Molecular Structure, vol. 935, no. 1-3, pp. 110-114, 2009.

[26] J. Dai and R. J. Mumper, "Plant phenolics: extraction, analysis and their antioxidant and anticancer properties," Molecules, vol. 15, no. 10, pp. 7313-7352, 2010.

[27] U. Landmesser, S. Spiekermann, C. Preuss et al., "Angiotensin II induces endothelial xanthine oxidase activation: role for endothelial dysfunction in patients with coronary disease," Arteriosclerosis, Thrombosis, and Vascular Biology, vol. 27, no. 4, pp. 943-948, 2007.

[28] K. Erdmann, N. Grosser, K. Schipporeit, and H. Schröder, "The ACE inhibitory dipeptide Met-Tyr diminishes free radical formation in human endothelial cells via induction of heme oxygenase-1 and ferritin," Journal of Nutrition, vol. 136, no. 8, pp. 2148-2152, 2006. 
[29] S. S. Raychaudhuri and X. W. Deng, "The role of superoxide dismutase in combating oxidative stress in higher plants," Botanical Review, vol. 66, no. 1, pp. 89-98, 2000.

[30] M. G. Miguel, "Anthocyanins: antioxidant and/or antiinflammatory activities," Journal of Applied Pharmaceutical Science, vol. 1, no. 6, pp. 7-15, 2011.

[31] M. P. Kähkönen and M. Heinonen, "Antioxidant activity of anthocyanins and their aglycons," Journal of Agricultural and Food Chemistry, vol. 51, no. 3, pp. 628-633, 2003.

[32] L. R. Fukumoto and G. Mazza, "Assessing antioxidant and prooxidant activities of phenolic compounds," Journal of Agricultural and Food Chemistry, vol. 48, no. 8, pp. 3597-3604, 2000. 


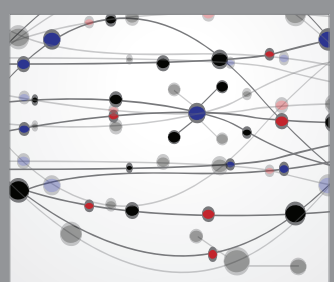

The Scientific World Journal
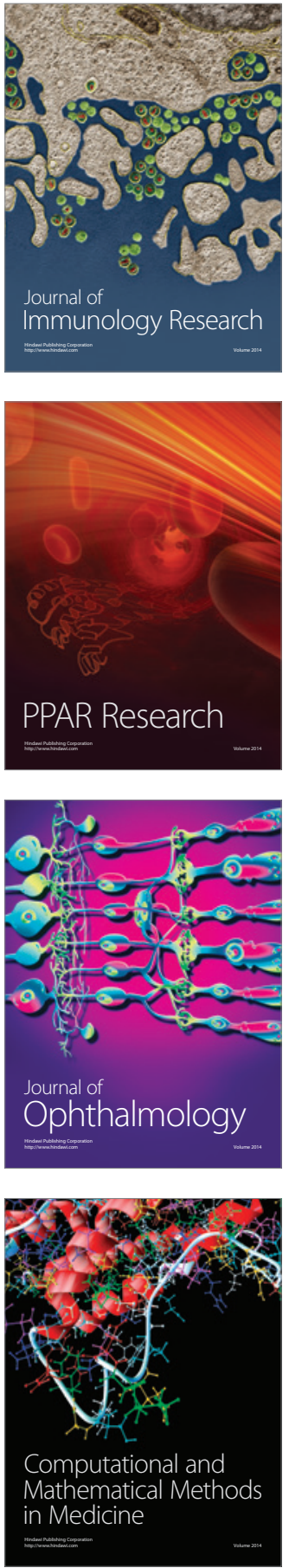

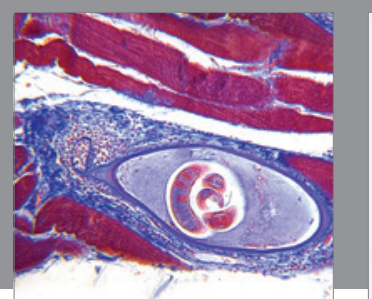

Gastroenterology Research and Practice

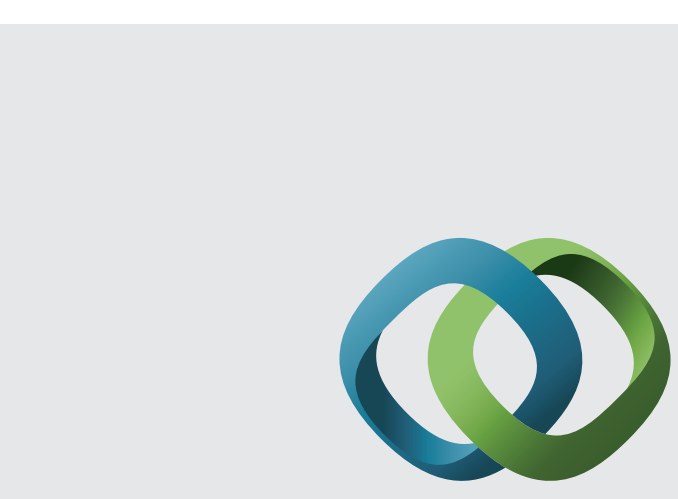

\section{Hindawi}

Submit your manuscripts at

http://www.hindawi.com
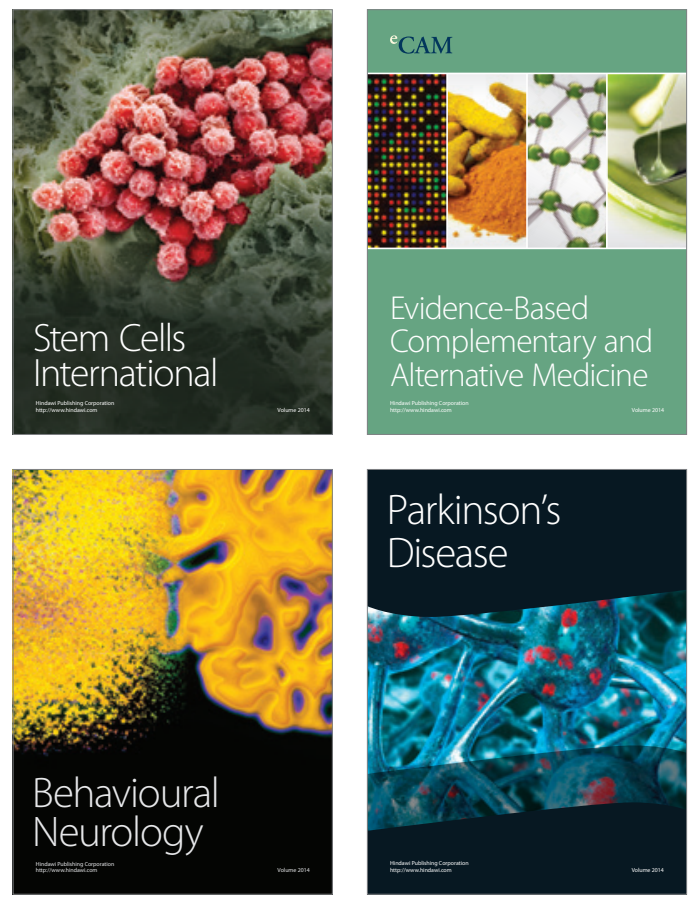
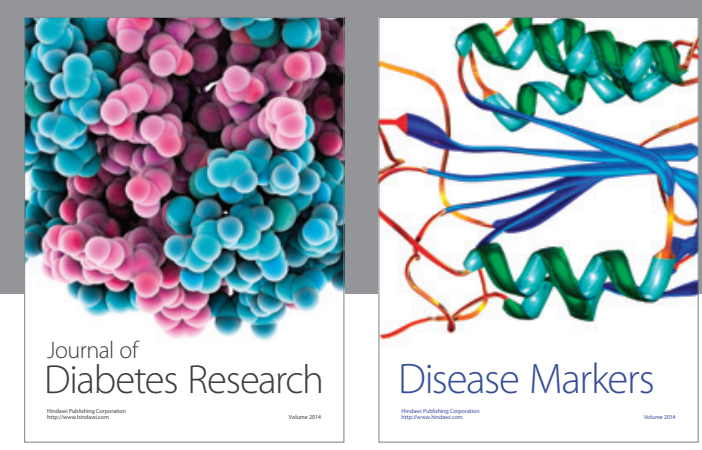

Disease Markers
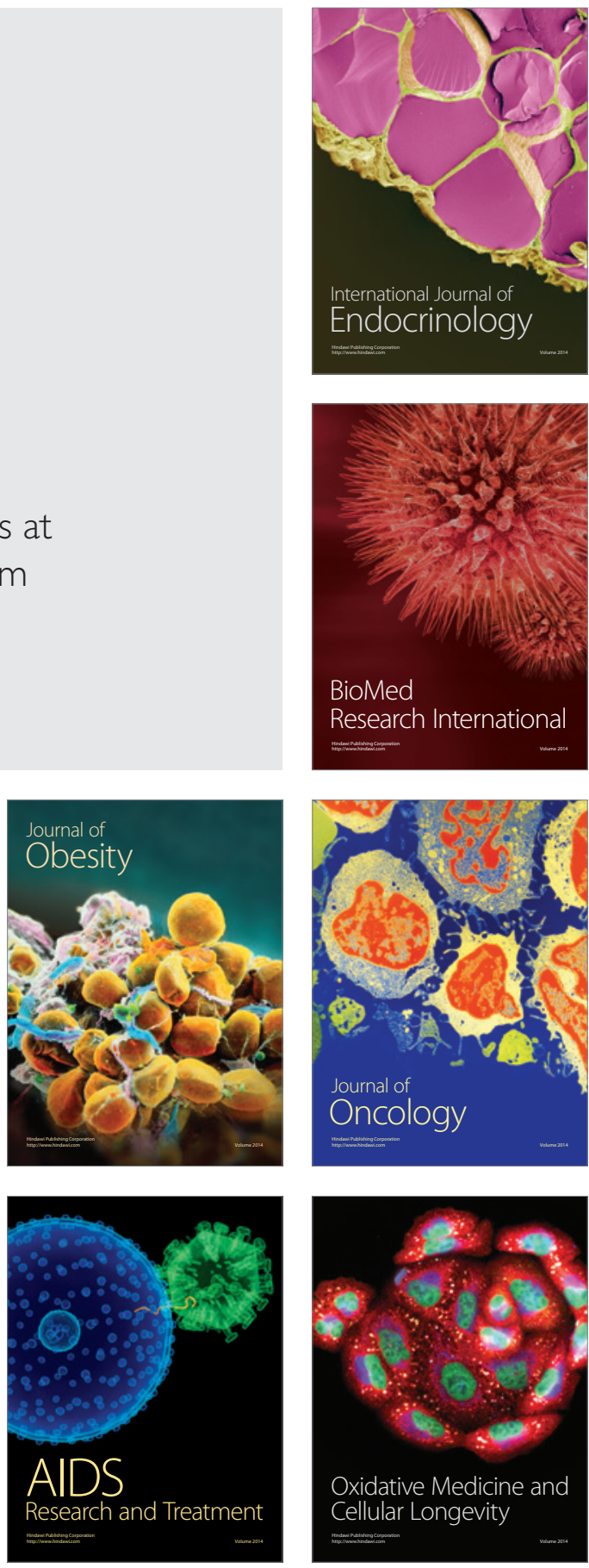